\title{
Avaliação do conhecimento de jovens universitários quanto às infecções sexualmente
}

\section{transmissíveis}

\author{
Assessment of the knowledge of university young people regarding sexually transmitted infections \\ Evaluación del conocimiento de los jóvenes universitarios sobre las infecciones de transmisión
}

sexual

Recebido: 09/07/2021 | Revisado: 18/07/2021 | Aceito: 19/07/2021 | Publicado: 26/07/2021

\author{
Lara Evangelista Orlandi \\ ORCID: https://orcid.org/0000-0003-3926-0750 \\ Centro Universitário de Lavras, Brasil \\ E-mail: laraorlandi@hotmail.com \\ Adriano Rodrigues \\ ORCID: https://orcid.org/0000-0003-3312-2306 \\ Centro Universitário de Lavras, Brasil \\ E-mail: adrianorodrigues@unilavras.edu.br \\ Natália Galvão Garcia \\ ORCID: https://orcid.org/0000-0002-1659-8524 \\ Centro Universitário de Lavras, Brasil \\ E-mail: nataliagalvao@unilavras.edu.br
}

\begin{abstract}
Resumo
De acordo com a Organização Mundial de Saúde (OMS) diariamente ocorrem cerca 1 milhão de novas infecções sexualmente transmissíveis (ISTs). Contudo, estas são consideradas um problema de saúde pública de âmbito mundial. No Brasil, casos de ISTs têm crescido, sobretudo entre os jovens. Sendo assim, este estudo teve como objetivo avaliar o nível de conhecimento de jovens universitários sobre as ISTs. O universo da pesquisa foi composto por 154 jovens entre 18 a 29 anos, os quais responderam a um questionário online e auto aplicado. Foram disponibilizados, uma cartilha e um folder, aos participantes com intuito de orientá-los sobre as ISTs. Na maioria das questões foi observado conhecimento geral dos participantes. No entanto, em relação às formas de transmissão indiretas, os participantes mostraram falta de conhecimento quando comparadas às formas de transmissão direta, as quais são mais divulgadas. Assim como também foi observado um obscurantismo quanto à algumas formas de manifestação clínica, e algumas ISTs. Além disso, uma parcela significativa dos participantes demonstrou constrangimento ao falar sobre o assunto. E apesar, da maioria afirmar estar segura para a vida sexual com o conhecimento prévio, grande parte dos participantes afirmou que gostaria de receber mais informações sobre as ISTs.
\end{abstract}

Palavras-chave: Infecções sexualmente transmissíveis; Conhecimento; Adolescentes.

\begin{abstract}
According to the World Health Organization (WHO), about 1 million new sexually transmitted infections (STIs) occur daily. However, these are considered a worldwide public health problem. In Brazil, cases of STIs have grown, especially among young people. Therefore, this study aimed to assess the level of knowledge of young university students about STIs. The research universe consisted of 154 young people aged between 18 and 29 years old, who answered an online and self-applied questionnaire. A booklet and a folder were made available to participants to guide them about STIs. In most questions, general knowledge of the participants was observed. However, regarding indirect forms of transmission, participants showed a lack of knowledge when compared to forms of direct transmission, which are more publicized. As well as an obscurantism regarding some forms of clinical manifestation, and some STIs. In addition, a significant portion of the participants showed embarrassment when talking about the subject. And despite the majority claiming to be safe for sex life with prior knowledge, most participants said they would like to receive more information about STIs. Keywords: Sexually transmitted diseases; Knowledge; Adolescents.
\end{abstract}

\section{Resumen}

Según la Organización Mundial de la Salud (OMS), diariamente ocurren alrededor de 1 millón de nuevas infecciones de transmisión sexual (ITS). Sin embargo, estos se consideran un problema de salud pública mundial. En Brasil, los casos de ITS han aumentado, especialmente entre los jóvenes. Por tanto, este estudio tuvo como objetivo evaluar el nivel de conocimiento de los jóvenes universitarios sobre las ITS. El universo de investigación estuvo formado por 154 jóvenes de entre 18 y 29 años, que respondieron un cuestionario online y autoaplicado. Se puso a disposición de los 
participantes un folleto y una carpeta para orientarlos sobre las ITS. En la mayoría de las preguntas se observó un conocimiento general de los participantes. Sin embargo, con respecto a las formas de transmisión indirecta, los participantes mostraron un desconocimiento en comparación con las formas de transmisión directa, que son más publicitadas. Así como un oscurantismo con respecto a algunas formas de manifestación clínica y algunas ITS. Además, una parte importante de los participantes mostró vergüenza al hablar sobre el tema. Y a pesar de que la mayoría afirma ser segura para la vida sexual con conocimientos previos, la mayoría de los participantes dijeron que les gustaría recibir más información sobre las ITS.

Palabras clave: Infecciones de transmisión sexual; Conocimiento; Adolescentes.

\section{Introdução}

As Infecções Sexualmente Transmissíveis (ISTs), antigamente chamadas de Doenças Sexualmente Transmissíveis englobam uma variedade de patologias provocadas por vírus, bactérias e outros microrganismos, as quais podem ser disseminadas pelas diferentes formas de contato sexual (vaginal, anal e oral) quando desprotegidas. Além disso, mesmo que em menor proporção, as ISTs também podem ser transmitidas por vias indiretas, como pelo contato com secreções corporais contaminadas (Bruce \& Rogers, 2004; Belda, Shiratsu, \& Pinto, 2009; Decker, 2016).

De acordo com o último Boletim da Organização Mundial de Saúde (OMS) diariamente ocorrem cerca de 1 milhão de novas infecções. Sendo, as ISTs consideradas um problema de saúde pública de âmbito mundial (Workowski \& Bolan, 2015; Decker, 2016).

No Brasil, casos de infecções sexualmente transmissíveis (ISTs) têm crescido, sobretudo entre os jovens de 15 a 29 anos. Entre as mais comuns estão a clamídia, gonorreia, sífilis, tricomoníase, HPV, hepatites virais e HIV. Entre 2010 e 2018 , os diagnósticos de sífilis adquirida sexualmente aumentaram em 3.600\% no país e a AIDS se tornou uma epidemia entre os jovens, pois, mantém um nível crescente nos indivíduos abaixo 35 anos (Brasil, 2018).

Todas as pessoas possuem risco de infecção, porém há uma prevalência significativa na população jovem, homossexuais e uma minoria racial e étnica, sendo os jovens responsáveis por quase metade de todos os casos relatados anualmente (Decker, 2016; Sanz-lorente, Wanden-Berghe, \& Castejón-Bolea, 2018; Tributino et al., 2018). Além disso, há pesquisas que indicam que jovens com menor poder aquisitivo, muitas vezes abandonam os estudos para trabalharem, o que consequentemente gera um déficit de conhecimento em relação às ISTs (Vilella \& Doretto, 2006).

Especialistas apontam que essa geração possui um maior risco de contrair ISTs devido a diferentes aspectos como: desconhecimento sobre as formas de prevenção; descrença nessas doenças; sexualidade iniciada de forma prematura; multiplicidade de parceiros, comportamentos de risco, como o uso de bebidas alcoólicas e drogas, entre outros. Ademais, esses fatores também contribuem para outros problemas sociais nessa faixa etária (Romero et al., 2006; Camargo \& Ferrari, 2009; Castro, Araújo, \& Pitangui, 2017; Doubova, 2017; Genz et al., 2017; Kumar et al., 2018).

As ISTs podem resultar em muitas sequelas reprodutivas em longo prazo, incluindo infertilidade, gravidez, complicações, aumento do risco de aquisição do HIV e câncer. Sendo assim, levando em conta que os números de disseminação de ISTs têm aumentado cada dia mais, as medidas gerais de promoção e educação em saúde têm se mostrado essenciais na prevenção dessas doenças, pois trazem informações e trocas de experiências que colaboram para o aumento de informações direcionadas a percepção dos fatores de risco, além de promover a prática sexual segura pela mudança de comportamento e adoção de preservativos (Bottega et al., 2016; Sanz-lorente, Wanden-Berghe, \& Castejón-Bolea, 2018).

Essas ações educativas também atuam diretamente sobre a importância da detecção dos sinais e sintomas, dos métodos de transmissão, do diagnóstico e do tratamento, promovendo um controle mais eficiente. Pois, o acesso às informações pode melhorar a capacidade do reconhecimento das ISTs, aumentando a procura por atendimento (Villela \& Doretto, 2006; Beserra et al., 2008; Sanz-lorente, Wanden-Berghe, \& Castejón-Bolea, 2018). 
Com base nesses aspectos, este estudo teve como objetivo avaliar o nível de conhecimento e o comportamento de jovens universitários de diferentes áreas sobre as infecções sexualmente transmitidas.

\section{Metodologia}

Este estudo foi submetido à apreciação do Comitê de Ética em Pesquisa em Seres Humanos do Centro Universitário de Lavras - UNILAVRAS e aprovado pelo mesmo (CAAE:37946620.0.0000.5116). Os dados foram coletados por meio de questionários on-line e auto aplicado, no qual inicialmente o voluntário teve acesso ao Termo de Consentimento Livre e Esclarecido - TCLE e após a sua leitura pôde optar por participar ou não da pesquisa, assinalando sua opção antes do início da coleta de dados.

O universo da pesquisa foi composto por jovens que preencheram os critérios de inclusão, sendo eles: ter de 18 a 29 anos, ser aluno de graduação e aceitarem participar da pesquisa. Tratou-se de uma amostra por conveniência, pela facilidade de acesso aos sujeitos do estudo. De acordo com a metodologia científica, a natureza da pesquisa é quantitativa (Estrela, 2018).

Ao final do questionário disponibilizou-se uma cartilha e um folder aos participantes com intuito de orientá-los sobre as infecções sexualmente transmissíveis.

As respostas coletadas nos questionários foram tabuladas formando um banco de dados. A análise foi realizada, basicamente, através de estatística descritiva para a caracterização da amostra, seguida de teste para proporções - Teste Exato de Fisher - a fim de comparar se as proporções dos que responderam sim entre as mulheres diferiam da mesma proporção entre os homens, sendo considerado o nível de significância de 5\%.

\section{Resultados}

A amostra deste estudo foi constituída por 154 universitários, dos quais 69,6\% eram do sexo feminino e 30,4\% do sexo masculino. A idade média dos participantes foi de 22,3 anos, com intervalo de confiança de $(22,3 \pm 0,3)$ anos. Com relação ao estado civil, a maioria era solteiro $(98,7 \%)$, sendo os demais casados $(0,65 \%)$ ou divorciados $(0,65 \%)$. A origem dos universitários foi composta por $57,8 \%$ da área da saúde e $42,2 \%$ provindos de cursos de outras áreas.

Dentre os participantes, 82,6\% afirmavam ter iniciado a vida sexual entre 15 e 20 anos, 8,7\% entre 20 e $25,7,5 \%$ com menos de 15 anos e 1,2\% entre 25 e 29 anos como pode ser observado na Figura 1.

Figura 1 - Distribuição dos participantes de acordo com a faixa etária de início da vida sexual.

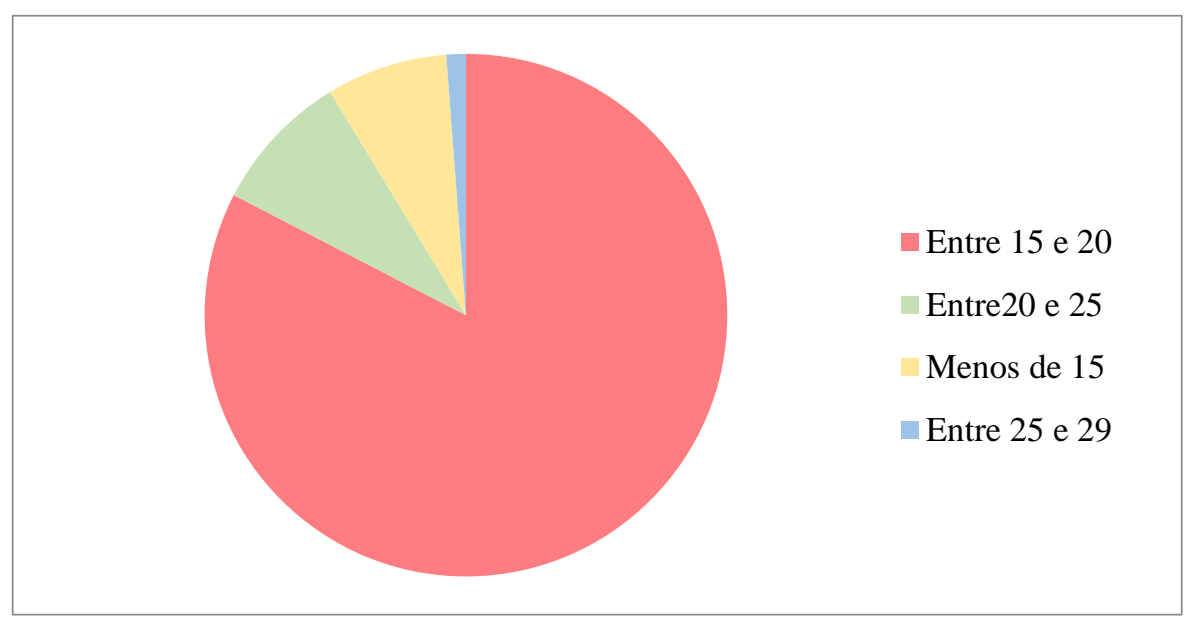

Fonte: Dados da pesquisa. 
Research, Society and Development, v. 10, n. 9, e31210918077, 2021

(CC BY 4.0) | ISSN 2525-3409 | DOI: http://dx.doi.org/10.33448/rsd-v10i9.18077

Em relação ao número de parceiros, a maior parte (30,4\%) afirmou ter tido até 5 parceiros, seguida por $24,8 \%$ que afirmaram terem tido apenas um parceiro, $19,3 \%$ entre 5 e 10, 17,4\% afirmarem terem tido mais de 10 parceiros e $8,1 \%$ preferiram não responder (Figura 2).

Figura 2 - Distribuição dos participantes de acordo com o número de parceiros sexuais.

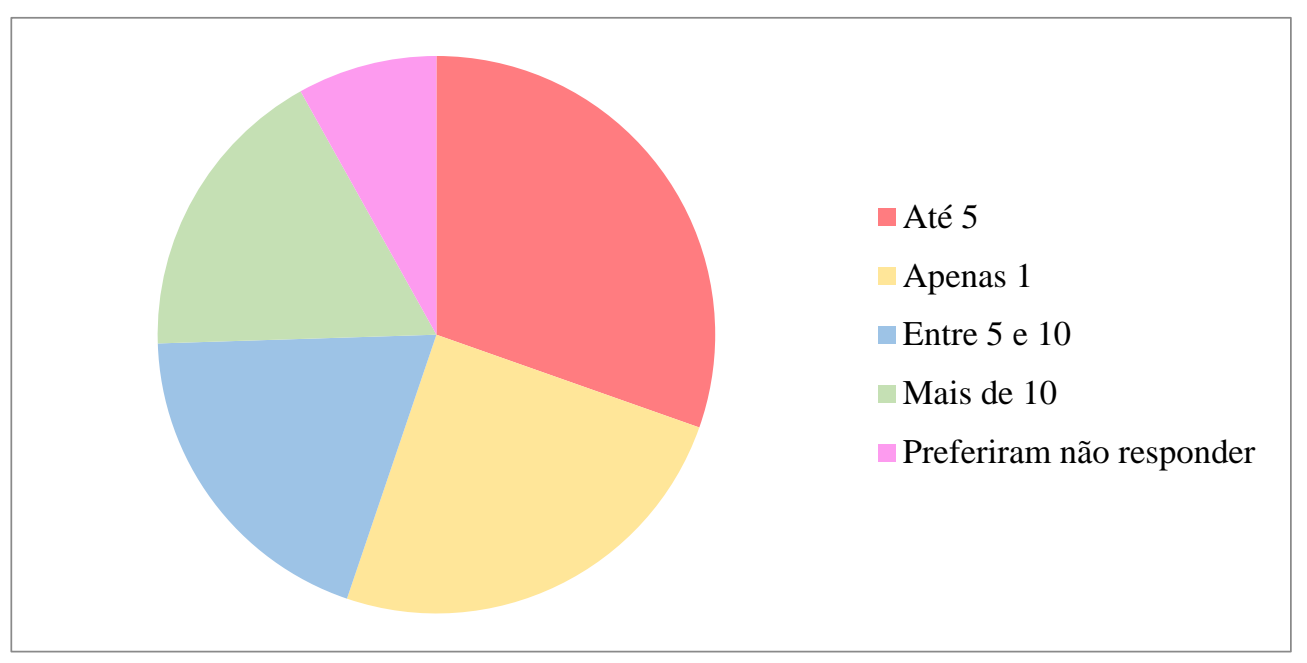

Fonte: Dados da pesquisa.

Apesar de quase a totalidade da amostra $(99,4 \%)$ ter afirmado considerar importante o uso de preservativos, $88,8 \%$ afirmaram já terem tido relação sexual de forma desprotegida. Além disso, apenas 51,6\% afirmaram já terem feito teste para ISTs.

Após as perguntas de caracterização demográfica, o conhecimento dos participantes acerca das ISTs foi avaliado por meio de questões com respostas positivas ou negativas como mostra a tabela abaixo (Tabela 1). Na maioria das questões foi observado conhecimento geral dos participantes. No entanto, em relação às formas de transmissão indiretas, como por exemplo, pelo uso de agulhas, pelo beijo, pelo toque físico, entre outras, os participantes mostraram falta de conhecimento quando comparadas às formas de transmissão por via direta, as quais são mais divulgadas (Figura 3). 
Tabela 1 - Comparação do conhecimento acerca das infecções sexualmente transmissíveis entre mulheres e homens.

\begin{tabular}{|c|c|c|c|c|c|}
\hline & \multicolumn{2}{|c|}{ Mulheres } & \multicolumn{2}{|c|}{ Homens } & \multirow{2}{*}{ Valor $p$} \\
\hline & Sim & Não & Sim & Não & \\
\hline Sabe o que é IST & $\begin{array}{c}111 \\
(99,1 \%)\end{array}$ & $\begin{array}{c}1 \\
(0,9 \%)\end{array}$ & $\begin{array}{c}40 \\
(95,2 \%)\end{array}$ & $\begin{array}{c}2 \\
(4,8 \%)\end{array}$ & 1,0000 \\
\hline Sabe se prevenir de IST & $\begin{array}{c}109 \\
(97,3 \%)\end{array}$ & $\begin{array}{c}2 \\
(2,7 \%)\end{array}$ & $\begin{array}{c}42 \\
(100 \%)\end{array}$ & $\begin{array}{c}0 \\
(0 \%)\end{array}$ & 1,0000 \\
\hline $\begin{array}{l}\text { Sabe que pode contrair IST por sexo } \\
\text { vaginal }\end{array}$ & $\begin{array}{c}112 \\
(100 \%)\end{array}$ & $\begin{array}{c}0 \\
(0 \%)\end{array}$ & $\begin{array}{c}42 \\
(100 \%)\end{array}$ & $\begin{array}{c}0 \\
(0 \%)\end{array}$ & 1,0000 \\
\hline Sabe que pode contrair IST por sexo anal & $\begin{array}{c}112 \\
(100 \%) \\
\end{array}$ & $\begin{array}{c}0 \\
(0 \%) \\
\end{array}$ & $\begin{array}{c}42 \\
(100 \%) \\
\end{array}$ & $\begin{array}{c}0 \\
(0 \%) \\
\end{array}$ & 1,0000 \\
\hline Sabe que pode contrair IST por sexo oral & $\begin{array}{c}108 \\
(96,4 \%)\end{array}$ & $\begin{array}{c}4 \\
(3,6 \%)\end{array}$ & $\begin{array}{c}41 \\
(97,6 \%)\end{array}$ & $\begin{array}{c}1 \\
(2,4 \%)\end{array}$ & 1,0000 \\
\hline $\begin{array}{l}\text { Sabe que pode contrair IST pelo } \\
\text { compartilhamento de agulhas no uso de } \\
\text { drogas injetáveis }\end{array}$ & $\begin{array}{c}110 \\
(98,2 \%)\end{array}$ & $\begin{array}{c}2 \\
(1,8 \%)\end{array}$ & $\begin{array}{c}39 \\
(92,9 \%)\end{array}$ & $\begin{array}{c}3 \\
(7,1 \%)\end{array}$ & 0,1254 \\
\hline $\begin{array}{l}\text { Sabe que pode contrair IST pelo } \\
\text { compartilhamento de objetos perfuro- } \\
\text { cortantes como alicates, pinças e tesouras }\end{array}$ & $\begin{array}{c}106 \\
(94,6 \%)\end{array}$ & $\begin{array}{c}6 \\
(5,4 \%)\end{array}$ & $\begin{array}{c}37 \\
(88,1 \%)\end{array}$ & $\begin{array}{c}5 \\
(11,9 \%)\end{array}$ & 0,1723 \\
\hline $\begin{array}{l}\text { Sabe que pode contrair uma IST pelo } \\
\text { contato com saliva, beijo, uso de talheres }\end{array}$ & $\begin{array}{c}36 \\
(32,1 \%)\end{array}$ & $\begin{array}{c}76 \\
(67,9 \%)\end{array}$ & $\begin{array}{c}23 \\
(54,8 \%)\end{array}$ & $\begin{array}{c}19 \\
(45,2 \%)\end{array}$ & $0,0150^{*}$ \\
\hline $\begin{array}{l}\text { Sabe que é possível contrair uma IST } \\
\text { através do toque físico }\end{array}$ & $\begin{array}{c}14 \\
(12,5 \%)\end{array}$ & $\begin{array}{c}98 \\
(87,5 \%)\end{array}$ & $\begin{array}{c}6 \\
(14,3 \%)\end{array}$ & $\begin{array}{c}36 \\
(85,7 \%)\end{array}$ & 0,7905 \\
\hline $\begin{array}{l}\text { Considera importante o uso de } \\
\text { preservativos }\end{array}$ & $\begin{array}{c}112 \\
(100 \%)\end{array}$ & $\begin{array}{c}0 \\
(0 \%)\end{array}$ & $\begin{array}{c}41 \\
(97,6 \%)\end{array}$ & $\begin{array}{c}1 \\
(2,4 \%)\end{array}$ & 0,2727 \\
\hline
\end{tabular}

* Significativo a 5\% de significância. Fonte: Dados da pesquisa (2021).

Figura 3 - Distribuição das formas de transmissão diretas e indiretas.

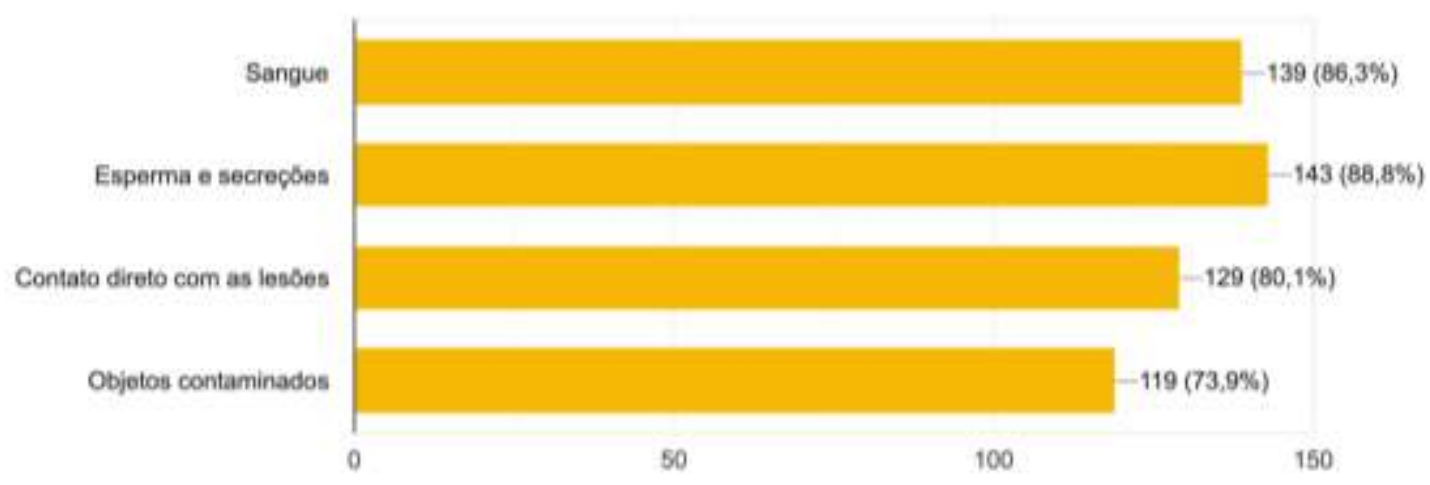

Fonte: Dados da pesquisa (2021).

Com base nos resultados obtidos foi possível inferir que apenas as proporções relativas ao conhecimento sobre a possibilidade de contrair uma IST pelo contato com saliva, beijo e/ou uso de talheres foram estatisticamente significativas entre os homens e as mulheres. Neste caso, a proporção de mulheres que possui este conhecimento $(32,1 \%)$ era estatisticamente menor que a proporção de homens com o mesmo conhecimento $(54,8 \%)(p=0,0150)$ (Tabela 1). Nas demais questões não foram observadas diferenças significativas entre a proporção de homens e mulheres. 
Quando questionados sobre as manifestações clínicas envolvidas, 99,3\% assinalaram a opção "corrimento ou sangramento" como sendo um tipo de manifestação mais comumente encontrada. Outras manifestações como mau cheiro, coceira e lesões avermelhadas ou esbranquiçadas também foram apontadas pela maioria dos participantes. Entretanto, a opção "lesões endurecidas" mostrou ser a menos conhecida.

Dentre as infecções sexualmente transmissíveis avaliadas, a clamídia foi a IST que teve maior divergência de respostas, $80,5 \%$ responderam 'Sim", enquanto $19,5 \%$ responderam "Não", consequentemente indicando maior falta de conhecimento dos participantes. Seguida pelas hepatites virais que também apresentou respostas divergentes, cerca de 7,1\% dos participantes mostraram não ter conhecimento sobre a mesma (Tabela 2).

Com base nos resultados obtidos, estatisticamente não foram observadas diferenças significativas entre as proporções de homens e mulheres no que se refere ao conhecimento sobre as ISTs investigadas.

Ao serem indagados quanto aos meios que teriam recebido informações, as redes sociais, conversa com amigos e colégio foram os mais citados (Figura 4). No entanto, cerca de 23,6\% mostraram ter constrangimento de falar sobre o assunto.

Apesar da maioria afirmar se sentir segura para a vida sexual com o conhecimento prévio, $82 \%$ afirmaram que gostariam de receber mais informações sobre as infecções sexualmente transmissíveis.

Tabela 2 - Comparação do conhecimento acerca das infecções sexualmente transmissíveis entre mulheres e homens.

\begin{tabular}{|c|c|c|c|c|c|}
\hline & \multicolumn{2}{|c|}{ Mulheres } & \multicolumn{2}{|c|}{ Homens } & \multirow{2}{*}{ Valor $p$} \\
\hline & Sim & Não & Sim & Não & \\
\hline Já ouviu falar em AIDS & $\begin{array}{c}112 \\
(100 \%)\end{array}$ & $\begin{array}{c}0 \\
(0 \%)\end{array}$ & $\begin{array}{c}42 \\
(100 \%)\end{array}$ & $\begin{array}{c}0 \\
(0 \%)\end{array}$ & 1,0000 \\
\hline Já ouviu falar em Gonorreia & $\begin{array}{c}111 \\
(99,1 \%)\end{array}$ & $\begin{array}{c}1 \\
(0,9 \%)\end{array}$ & $\begin{array}{c}42 \\
(100 \%)\end{array}$ & $\begin{array}{c}0 \\
(0 \%)\end{array}$ & 1,0000 \\
\hline Já ouviu falar em Sífilis & $\begin{array}{c}112 \\
(100 \%)\end{array}$ & $\begin{array}{c}0 \\
(0 \%)\end{array}$ & $\begin{array}{c}42 \\
(100 \%)\end{array}$ & $\begin{array}{c}0 \\
(0 \%)\end{array}$ & 1,0000 \\
\hline Já ouviu falar em HPV & $\begin{array}{c}112 \\
(100 \%)\end{array}$ & $\begin{array}{c}0 \\
(0 \%)\end{array}$ & $\begin{array}{c}42 \\
(100 \%)\end{array}$ & $\begin{array}{c}0 \\
(0 \%)\end{array}$ & 1,0000 \\
\hline Já ouviu falar em Clamídia & $\begin{array}{c}90 \\
(80,4 \%)\end{array}$ & $\begin{array}{c}22 \\
(19,6 \%)\end{array}$ & $\begin{array}{c}34 \\
(81 \%)\end{array}$ & $\begin{array}{c}8 \\
(19 \%)\end{array}$ & 1,0000 \\
\hline Já ouviu falar em Herpes & $\begin{array}{c}112 \\
(100 \%)\end{array}$ & $\begin{array}{c}0 \\
(0 \%)\end{array}$ & $\begin{array}{c}42 \\
(100 \%)\end{array}$ & $\begin{array}{c}0 \\
(0 \%)\end{array}$ & 1,0000 \\
\hline Já ouviu falar em Hepatites Virais & $\begin{array}{c}102 \\
(91,1 \%)\end{array}$ & $\begin{array}{c}10 \\
(8,9 \%)\end{array}$ & $\begin{array}{c}41 \\
(97,6 \%)\end{array}$ & $\begin{array}{c}1 \\
(2,4 \%)\end{array}$ & 0,2907 \\
\hline
\end{tabular}

Fonte: Dados da pesquisa (2021). 
Figura 4 - Distribuição dos participantes quanto ao meio que teriam recebido.

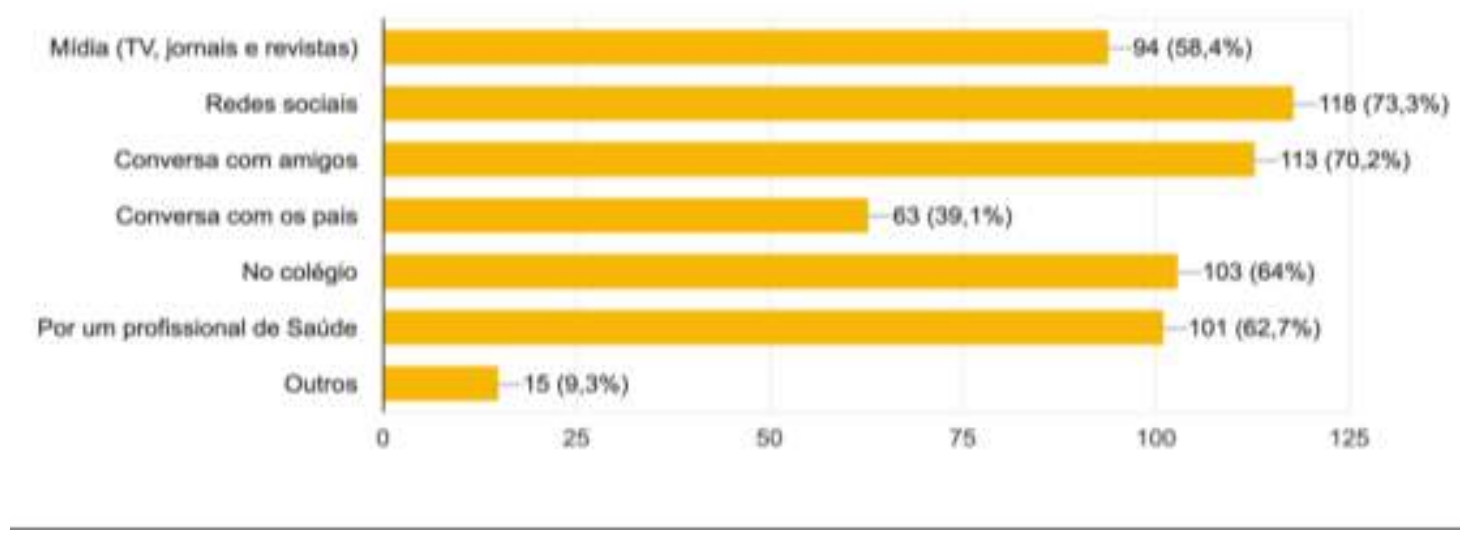

Fonte: Dados da pesquisa (2021).

\section{Discussão}

Apesar de atualmente, a maioria das pessoas ter fácil acesso à informação, o número de casos de infecções sexualmente transmissíveis (ISTs) têm crescido de forma exponencial nos últimos anos, fazendo com que as ISTs sejam consideradas um problema de saúde pública mundial (Workowski \& Bolan, 2015; Decker, 2016; Brasil, 2018).

Estudos recentes apontam que há uma prevalência significativa de ISTs entre os jovens. O que segundo eles pode estar ligado a diversos aspectos como o desconhecimento sobre as formas de prevenção; a descrença nessas doenças; a sexualidade iniciada de forma prematura; multiplicidade de parceiros, comportamentos de risco, entre outros (Camargo \& Ferrari, 2009; Castro, Araújo, \& Pitangui, 2017; Doubova, 2017; Genz et al., 2017; Kumar et al., 2018; Sanz-lorente, Wanden-Berghe, \& Castejón-Bolea, 2018; Tributino et al., 2018).)

Acredita-se que com o avanço da tecnologia, os jovens passaram a obter informações e conhecimentos com maior facilidade. No entanto, os assuntos sexualidade e ISTs ainda são pouco comentados, pois, uma parcela significativa dos jovens apresenta certo constrangimento (Garbin, et al., 2010), como foi observado no presente estudo, no qual cerca de 23,6\% dos participantes mostraram se sentirem constrangidos ao falarem sobre o assunto.

Quando se avalia o interesse na busca de conhecimento para se protegerem diante das ISTs, alguns autores sugerem haver um destaque das mulheres em relação aos homens (Gerhardt, Nader, \& Pereira, 2008). No entanto, com base nos resultados obtidos, estatisticamente não foram observadas diferenças significativas entre as proporções de homens e mulheres no que se refere ao conhecimento sobre as ISTs.

A maioria dos estudos mostra que as formas de transmissão direta via contato sexual (vaginal, oral e anal) são conhecidas por grande parte dos indivíduos (Camargo \& Ferrari, 2009; Decker, 2016; Castro, Araújo, \& Pitangui, 2017). Por outro lado, alguns autores destacam que as transmissões indiretas precisam ter um destaque maior (Spindola et al., 2019). Corroborando com estes autores, de acordo com os resultados obtidos no presente estudo, os participantes mostraram falta de conhecimento quanto às formas de transmissão por via indireta. Além disso, vale ressaltar que as proporções relativas ao conhecimento sobre a possibilidade de contrair uma IST pelo contato com saliva, beijo e/ou uso de talheres apontaram que o número de mulheres que possui este conhecimento $(32,1 \%)$ é estatisticamente menor que o número de homens com o mesmo conhecimento (54,8\%).

Clinicamente as ISTs podem se apresentar de diversas formas, todavia, os sinais mais conhecidos pelos jovens nesse estudo foram: corrimento, sangramento, mau cheiro, coceira e lesões avermelhadas ou esbranquiçadas. 
Alguns autores sugerem que os homens iniciam, a vida sexual mais cedo e têm um número maior de parceiros quando comparados às mulheres. No presente estudo, apesar de não terem sido feitas as avaliações de acordo com o gênero, vale ressaltar que dentre os participantes, $82,6 \%$ afirmavam ter iniciado a vida sexual entre 15 e 20 e a maior parte $(30,4 \%)$ afirmou ter tido até 5 parceiros.

Dentre as diferentes ISTs, as mais conhecidas são Aids, sífilis e herpes (Gerhardt, Nader, \& Pereira, 2008; Garbin et al., 2010; Spindola et al., 2019). Mas segundo Spindola et al (2019) ainda existe uma defasagem quanto ao conhecimento sobre ISTs como gonorreia e hepatite. Os resultados obtidos nesse estudo corroboram com os autores, pois, foi identificada uma divergência entre os participantes com relação a hepatite. Entretanto, a clamídia foi a IST que indicou maior falta de conhecimento dos participantes.

Atualmente, a camisinha é considerada o método preventivo de primeira escolha devido ao seu baixo custo, fácil acesso, popularidade e segurança (Camargo \& Ferrari, 2009; Decker, 2016; Castro, Araújo, \& Pitangui, 2017).

E assim como visto nos resultados obtidos, a grande maioria da população considera importante o uso de preservativos. Mas quando se trata da sua utilização, levantamentos mais antigos mostram um número maior de indivíduos que afirmavam fazer uso do mesmo (Gerhardt, Nader, \& Pereira, 2008), quando comparados com estudos atuais (GARBIN, et al., 2010) e com os resultados observados neste estudo, os quais indicam que cerca de $80 \%$ dos jovens afirmam não fazerem uso do preservativo.

Um outro aspecto que vem mudando nos últimos anos é o meio de divulgação das informações, pois, observa-se que a aquisição de conhecimento sobre as ISTs ocorria predominantemente nas escolas (Gerhardt, Nader, \& Pereira, 2008; Garbin et al., 2010). Todavia, hoje em dia as redes sociais ocupam posição de destaque $(73,3 \%)$ como o meio mais utilizado para receber informações.

Apesar de toda facilidade de acesso às informações e da maioria dos jovens afirmar estar segura para a vida sexual com o conhecimento prévio, ainda é perceptível as lacunas a serem preenchidas com orientações relevantes sobre o assunto (Spindola et al., 2019). Além de, grande parte dos jovens, como observado no presente estudo (82\%), afirmar que gostaria de receber mais informações sobre as infecções sexualmente transmissíveis.

\section{Considerações Finais}

De modo geral, concluiu-se que a maioria dos jovens sabe o que é uma IST, bem como os meios de transmissão direta. No entanto, há uma falta de conhecimento sobre algumas vias de transmissão indireta, como por exemplo, pelo uso de agulhas, pelo beijo, pelo toque físico, entre outras. Assim como também foi observado um obscurantismo quanto à algumas formas de manifestação clínica, e algumas ISTs, como a clamídia e a hepatite.

Além disso, uma parcela significativa dos participantes demonstrou constrangimento ao falar sobre o assunto. E apesar, da maioria afirmar estar segura para a vida sexual com o conhecimento prévio, grande parte dos participantes afirmou que gostaria de receber mais informações sobre as ISTs. O que deixa claro, a necessidade de mais ações educacionais envolvendo o tema.

\section{Referências}

Almeida, S. M. C. G. (2012). Pacientes com Hepatite Crônica: Manifestações Bucais. Revista da Universidade Vale do Rio Verde, $10(1), 264-274$.

Belda, W., Shiratsu, R., \& Pinto, V. (2009). Abordagem nas Doenças Sexualmente Transmissíveis. Anais. Brasileiros de Dermatologia84(2):151-159, 2009.

Beserra, E. P., Pinheiro, P. N. C., Alves, M. D. S., \& Barroso, M. G. T. (2008). Adolescência e Vulnerabilidade às Doenças Sexualmente Transmissíveis: Uma Pesquisa Documental. DST - Jornal Brasileiro de Doenças Sexualmente Transmissíveis, 20(1):32-35, 2008.

Bottega, A., Canestrini, T., Rodrigues, M. A., Rampelotto, R.F., dos Santos, S. O., Silva, D. C., \& Horner R. (2016). Abordagem das Doenças Sexualmente Transmissíveis na Adolescência: revisão de literatura. Revista Saúde. 91-104. 
Research, Society and Development, v. 10, n. 9, e31210918077, 2021

(CC BY 4.0) | ISSN 2525-3409 | DOI: http://dx.doi.org/10.33448/rsd-v10i9.18077

Brasil. (2018). Ministério da Saúde. Secretaria de Vigilância em Saúde Departamento de DST, AIDS e Hepatites Virais. Boletim Epidemiológico Hepatites Virais. 49(53):72.

Bruce, A. J., \& Rogers, R. S. (2004). Oral Manifestations of Sexually Transmitted Diseases. Clinics in Dermatology, (22) 6:520-527.

Camargo, E. Á. I., \& Ferrari, R. A. P. (2009). Adolescentes: Conhecimentos Sobre Sexualidade Antes e Após a Participação em 2009. Ciên. \& Saúde Coletiva, 14(3):937-846.

Carneiro, R. F., da Silva, N. C., Alves, T. A., Albuquerque, D. O., de Brito, D. C., \& Oliveira, L. L. (2015). Educação sexual na adolescência: uma abordagem no contexto escolar. SANARE-Revista de Políticas Públicas, 14(1):104-108.

Castro, J. F. L., Araújo, R. C. de; \& Pitangui, A. C. R. (2017). Sexual Behavior and Practices of School Adolescents in Recife City, Brazil. J. Hum. Growth Dev, 27(2): 219-227.

Decker, C. F. (2016). Sexually Transmitted Diseases: An Overview. Disease-a- Month, 62(8): 258-259.

Doubova, S. V. (2017). Effects of an Internet-based Educational Intervention to Prevent High-risk Sexual Behavior in Mexican Adolescents. Health education research, 32(6):487-498, 2017.

Estrela, C. (2018). Metodologia Científica: Ciência, Ensino, Pesquisa. Artes Médicas, 2005, p. 158.

Garbin, C. A. S., Lima, D. P., A., Dossi, A. P., Arcieri, R. M., \& Rovida, T. A. S. (2010). Percepção de Adolescentes em Relação a Doenças Sexualmente Transmissíveis e Métodos Contraceptivos. DST - Jornal brasileiro de Doenças Sexualmente Transmissiveis, 19(1):227-238.

Genz, N., Meincke, S. M. K., Carret, M. L. V., Corrêa, A. C. L., \& Alves, C. N. (2017). Doenças Sexualmente Transmissíveis: Conhecimento e Comportamento Sexual De Adolescentes. Texto \& Contexto Enfermagem, 26(2).1-12.

Gerhardt, C. R., Nader, S. S., \& Pereira, D. N. (2008). Doenças Sexualmente Transmissíveis: conhecimento, atitudes e comportamento entre os adolescentes de uma escola pública. Rev Bras Med Fam e Com, 3(12):257-270

Kumar, D., Goel, N. K., Bakshi, R. K., Sharma, M. K., \& Ghosh, A. K. (2017). Sexual Behavior of Adolescent Students in Chandigarh and Their Perceptions Regarding Family Life Education. Journal of Family Medicine and Primary Care, 6(2):108-110.

Romero, K. T., Medeiros, E. H. G. R., Vitalle, M. S. S., \& Wehba, J. (2007). O Conhecimento das Adolescentes Sobre Questões Relacionadas ao Sexo. Revista da Associação Médica Brasileira, 53(1):9-14.

Sanz-Lorente, M., Wanden-Berghe, C., Castejón-Bolea, R. (2018). Web 2.0 Tools in the Prevention of Curable Sexually Transmitted Diseases: Scoping Review. Journal of medical Internet research, 20(3):113.

Spindola, T., Oliveira, C. S. R., Santana, R. R. C., Sodré, C. P., de Oliveira, N. L. N., \& Brochado, E. J. (2019). Práticas Sexuais, Conhecimento e Comportamento dos Universitários em Relação às Infecções Sexualmente Transmissíveis. Rev Fund Care Online, 11(5):1135-41.

Tributino, A., Montgomery, M. C., Bertrand, T., Marak, T., Almonte, A., van den Berg, J., John, K. St., Medina, M. M., Morse, A., \& Chan, P. A. (2018). Partner Notification Outcomes After Integration of an On-site Disease Intervention Specialist at a Sexually Transmitted Disease Clinic. PloSone, 13(3):e0194041.

Villela, W. V., \& Doretto, D. T. (2006). Sobre a Experiência Sexual dos Jovens. Cadernos de Saúde Pública, 22(11) 2467-2472.

Workowski, K. A., \& Bolan, G. A. (2015). Sexually Transmitted Diseases Treatment Guidelines. MMWR. Recommendations and Reports: Morbidity and Mortality weekly Report, 64(03): 1-13. 